\title{
Prognostic factors in breast cancer with extracranial oligometastases and the appropriate role of radiation therapy
}

\author{
Gyu Sang Yoo, MD, Jeong II Yu, MD, Won Park, MD, PhD, \\ Seung Jae Huh, MD, PhD, Doo Ho Choi, MD, PhD \\ Department of Radiation Oncology, Samsung Medical Center, Sungkyunkwan University School of Medicine, Seoul, Korea
}

\begin{abstract}
Purpose: To identify prognostic factors for disease progression and survival of patients with extracranial oligometastatic breast cancer (EOMBC), and to investigate the role of radiation therapy (RT) for metastatic lesions.

Materials and Methods: We retrospectively reviewed the medical records of 50 patients who had been diagnosed with EOMBC following standard treatment for primary breast cancer initially, and received RT for metastatic lesions, with or without other systemic therapy between January 2004 and December 2008. EOMBC was defined as breast cancer with five or less metastases involving any organs except the brain. All patients had bone metastasis (BM) and seven patients had pulmonary, hepatic, or lymph node metastasis. Median RT dose applied to metastatic lesions was 30 Gy (range, 20 to $60 \mathrm{~Gy}$ ).

Results: The 5-year tumor local control (LC) and 3-year distant progression-free survival (DPFS) rate were 66.1\% and 36.8\%, respectively. High RT dose ( $\left.\geq 50 \mathrm{~Gy}_{10}\right)$ was significantly associated with improved LC. The 5 -year overall survival (OS) rate was $49 \%$. Positive hormone receptor status, pathologic nodal stage of primary cancer, solitary BM, and whole-lesion RT (WLRT), defined as RT whose field encompassed entire extent of disease, were associated with better survival. On analysis for subgroup of solitary BM, high RT dose was significantly associated with improved LC and DPFS, shorter metastasis-to-RT interval ( $\leq 1$ month) with improved DPFS, and WLRT with improved DPFS and OS, respectively.

Conclusion: High-dose RT in solitary BM status and WLRT have the potential to improve the progression-free survival and OS of patients with EOMBC.
\end{abstract}

Keywords: Oligometastasis, Radiotherapy, Breast neoplasms

\section{Introduction}

Metastatic breast cancer (MBC) is generally considered an incurable disease. Advances in modern systemic therapies have led to improved overall survival (OS) of patients with MBC [1]. In particular, the development of new cytotoxic agents such as taxanes, vinorelbine, and capecitabine; new hormonal agents such as third-generation aromatase inhibitors; and target therapies such as trastuzumab and lapatinib have exhibited greater treatment efficacy and yielded improved OS of patients with MBC [2]. However, MBC is a heterogeneous disease and a subset of patients with MBC has particularly distinctive characteristics. This subset of patients, only $1 \%$ to $10 \%$ of all patients with MBC [3], shows some

Received 16 July 2015, Revised 29 Septmber 2015, Accepted 27 October 2015.

Correspondence: Doo Ho Choi, MD, PhD, Department of Radiation Oncology, Samsung Medical Center, Sungkyunkwan University School of Medicine, 81 Irwon-ro, Gangnam-gu, Seoul 06351, Korea. Tel: +82-2-3410-2436, Fax: +82-2-3410-2619, E-mail: doho.choi@samsung.com

(c) This is an Open Access article distributed under the terms of the Creative Commons Attribution Non-Commercial License (http://creativecommons.org/ licenses/by-nc/4.0/) which permits unrestricted non-commercial use, distribution, and reproduction in any medium, provided the original work is properly cited.

www.e-roj.org 
benefits in survival outcome by multidisciplinary therapies including local modalities. The disease status of the subset is potentially curable stage IV disease, which is represented by oligometastatic disease [4].

In 1995, Hellman and Weichselbaum [5] suggested a new clinically significant state of cancer spread referred to as oligometastases (OM). Oligometastatic states were proposed to be the disease states in which a limited number of metastases are present, but the facilities for metastatic growth are not fully developed. According to this paradigm, metastasis-directed therapies for the OM state can be curative. This hypothesis is supported by evidences favoring curative resections of pulmonary metastases from sarcomas and renal cell cancer, in addition to hepatic resections for metastatic colorectal cancer $[6,7]$. Since the concept of OM was proposed, a number of studies have shown curative effects of local therapies such as resection and ablative therapy, including radiation therapy $(\mathrm{RT})$ and radiofrequency ablation for metastases [8].

Like metastatic colorectal cancer and sarcomas, surgical resection for metastases in patients with $M B C$ is associated with an improved survival rate $[3,4,9]$. RT for metastases is also associated with improved OS in patients with MBC. With the introduction of stereotactic ablative body radiation (SABR), which yields prospective local control (LC) rates ranging from $67 \%$ to $95 \%$, the role of RT has recently changed from palliative to potentially curative for selected patients with $\operatorname{MBC}[8,10,11]$. Especially, the roles of surgery and radiosurgery have been proved by phase III randomized trials and various outcome analyses for intracranial metastasis [12-14]. However, the role of RT for patients with extracranial MBC, whose prognosis are quite different from intracranial $M B C$, has not been investigated sufficiently [15].

In this study, we evaluated the role of RT in treating patients with extracranial oligometastatic breast cancer (EOMBC) by analyzing potential prognostic factors. The aims of this study were to identify the subset of patients with EOMBC for whom local RT treatment of metastasis would be most beneficial and to determine the optimal RT properties for these patients.

\section{Materials and Methods}

\section{Patients}

We retrospectively reviewed the medical records of 230 patients who initially received standard treatments including surgery with neoadjuvant or adjuvant therapies for primary breast cancer and thereafter were diagnosed as MBC and received RT for metastatic lesions between January 2004 and December 2008. Among them, patients with EOMBC which was defined as $M B C$ with five or less metastases involving any organs except the brain were included as the study population. And also we excluded synchronous MBC patients and also those who initially were diagnosed as non-MBC initially, but did not receive standard treatment for primary breast cancer before metastasis. After application of these criteria, the final cohort was composed of 50 patients. Metastases were diagnosed by computed tomography (CT), magnetic resonance imaging (MRI), whole body bone scanning, and positron emission tomography. The sizes of the metastatic tumors were measured by CT or MRI.

\section{Treatment and evaluation}

After diagnosis of EOMBC, all patients in the study were treated with RT to metastatic lesion of interest, either with or without other local therapy or systemic therapy. Wholelesion RT (WLRT), defined as RT whose field encompassed the entire extent of metastatic disease, was applied to every case in which WLRT was feasible. Otherwise, the target of RT was limited to some portion of the metastatic lesion according to clinician's decision. Median RT dose was $30 \mathrm{~Gy}$ (range, 20 to $60 \mathrm{~Gy}$ ). Treatment responses were evaluated according to the Response Evaluation Criteria in Solid Tumors (RECIST) guidelines ver. 1.1 [16]. To measure changes in tumor size, follow-up CT and MRI scans were examined in time interval of 1 to 3 months after the end of RT, and for patients without any evidence of disease, the time interval was prolonged to every 6 month or every year. We defined local failure as progressive disease within the RT field as assessed by the revised RECIST criteria. Distant progression was defined as the emergence of new distant metastasis at a site other than that of the first metastasis.

\section{Statistical analyses and variables}

Fisher exact test and logistic regression were used to analyze the related factors. LC duration was calculated as the time interval from the start of RT to the first observation of local progression or the last imaging follow-up visit if no progression had occurred. Distant progression-free survival (DPFS) was defined as the time interval from the diagnosis of EOMBC to the first observation of distant progression or the last follow-up visit if no progression. OS time was defined as the time interval between the diagnosis of metastasis and death, or the last follow-up visit if the patient was alive. Metastasis-free interval (MFI) was defined as the time 
interval from the beginning of treatment for primary breast cancer to the first diagnosis of distant metastasis. We defined metastasis-to-RT interval (MRTI) as the time interval from the diagnosis of EOMBC to the start of RT to metastatic lesion of interest. Univariate analysis was performed with the KaplanMeier method and the log-rank test to compare LC, DPFS, and OS rates for various factors. A multivariate Cox proportional hazards regression model was applied to determine the adjusted effects of these factors. For all analyses, statistical significance was set at $p \leq 0.05$.

\section{Results}

\section{Patient characteristics}

Patient and treatment characteristics are given in Table 1. The median age at the initial diagnosis of metastasis was 43 years (range, 24 to 72 years); 49 patients were female and one patient was male. For primary breast cancer, four patients received neoadjuvant chemotherapy and 44 patients underwent adjuvant chemotherapy. Two patients did not receive any chemotherapy initially for the primary disease. Both these patients had T1N0 stage disease and positive hormone receptor (HR) status; therefore, endocrinologic therapies were considered to be enough to control the primary breast cancer. Twenty-seven patients were treated with an adriamycin-based regimen and 21 patients were treated with a non-adriamycinbased regimen. HR status was determined for all patients. Of the 40 patients with HR-positive status, 35 patients received adjuvant endocrinologic therapy for primary breast cancer. Human epidermal growth factor receptor 2 (HER2) status was available for 48 patients; none of the HER2-positive patients received trastuzumab therapy. All patients had bone metastasis (BM) and seven patients had extrabony metastases. Thirty-one patients had solitary metastasis, all of which were solitary BM. Thirty-five patients had symptoms, mostly pain, and the others were asymptomatic. Two patients received surgical treatment including tumor excision and corpectomy for metastatic lesions before RT. Twenty patients were treated with bisphosphonate. Twelve patients received palliative chemotherapy and thirtyfour patients did palliative endocrinologic therapy for control of EOMBC, respectively. Patients who showed progression of disease received other systemic therapies with switched regimens. The median duration of follow-up after the first diagnosis of EOMBC was 53.6 months (range, 0 to 108 months). The median MFI was 40 months (range, 4 to 122 months).
Table 1. Patient and treatment characteristics $(n=50)$

\begin{tabular}{|c|c|}
\hline Variable & No. of patients (\%) \\
\hline \multicolumn{2}{|l|}{ Sex } \\
\hline Female & 49 (98.0) \\
\hline Male & $1(2.0)$ \\
\hline \multicolumn{2}{|l|}{ pT stage } \\
\hline pT1 & $25(50.0)$ \\
\hline pT2-4 & $25(50.0)$ \\
\hline \multicolumn{2}{|l|}{ pN stage } \\
\hline pNO & $16(32.0)$ \\
\hline pN1 & $16(32.0)$ \\
\hline pN2-3 & $18(36.0)$ \\
\hline \multicolumn{2}{|c|}{ Hormone receptor status } \\
\hline Negative & $10(20.0)$ \\
\hline Positive & $40(80.0)$ \\
\hline \multicolumn{2}{|l|}{$\mathrm{HER} 2^{\text {a) }}$} \\
\hline Negative & $37(77.1)$ \\
\hline Positive & $11(22.9)$ \\
\hline \multicolumn{2}{|c|}{ No. of metastatic lesions } \\
\hline 1 & $31(62.0)$ \\
\hline$\geq 2$ & $19(38.0)$ \\
\hline \multicolumn{2}{|l|}{ Site of metastasis } \\
\hline Bone & $50(100)$ \\
\hline Liver & $1(2.0)$ \\
\hline Lung & $2(4.0)$ \\
\hline Lymph node & $4(8.0)$ \\
\hline \multicolumn{2}{|l|}{$\mathrm{RT}$ dose $\left(\mathrm{Gy}_{10}\right)$} \\
\hline$<50$ & $33(66.0)$ \\
\hline$\geq 50$ & $17(34.0)$ \\
\hline \multicolumn{2}{|c|}{ Palliative chemotherapy } \\
\hline Yes & $12(24.0)$ \\
\hline No & $38(76.0)$ \\
\hline \multicolumn{2}{|c|}{ Palliative endocrinologic therapy } \\
\hline Yes & $34(68.0)$ \\
\hline No & $16(32.0)$ \\
\hline
\end{tabular}

HER2, human epidermal growth factor receptor $2 ; \mathrm{RT}$, radiation therapy; Gy ${ }_{10}$, biological equivalent dose in Gy with $\alpha / \beta=10$. ${ }^{a}$ HER2 receptor status was abailable for only 48 patients.

\section{Progression patterns}

Data from 43 patients (86\%) were available for evaluation of the initial responses to RT. The 3-year and 5-year tumor $\mathrm{LC}$ rates were $69.6 \%$ and $66.1 \%$, respectively. The complete response (CR), partial response (PR), and stable disease (SD) rates were 6.9\% (3 cases), 18.6\% (8 cases), and 74.4\% (32 cases), respectively. No local progression was observed in any CR case, whereas $25 \%$ of PR cases and $31.3 \%$ of SD cases exhibited local progression. HER2-negative $(p=0.019)$ status and higher RT dose ( $\geq 50 \mathrm{~Gy}_{10}, \mathrm{p}=0.036$ ) were significantly associated with improved LC by univariate analysis (Table 2). On multivariate analysis, higher RT dose was identified as 
Table 2. Univariate analysis

\begin{tabular}{|c|c|c|c|c|c|c|}
\hline \multirow{2}{*}{ Variable } & \multicolumn{2}{|c|}{$5-y r$ LC } & \multicolumn{2}{|c|}{ 3-yr DPFS } & \multicolumn{2}{|c|}{$5-y r$ OS } \\
\hline & $\%$ & $p$-value & $\%$ & p-value & $\%$ & $p$-value \\
\hline Age (yr) & & 0.611 & & 0.480 & & 0.293 \\
\hline$<40$ & 71.3 & & 43.4 & & 53.5 & \\
\hline$\geq 40$ & 63.3 & & 32.3 & & 46.2 & \\
\hline Hormone receptor & & 0.924 & & 0.938 & & 0.097 \\
\hline Negative & 66.7 & & 36.0 & & 40.0 & \\
\hline Positive & 66.2 & & 37.3 & & 51.1 & \\
\hline HER2 & & 0.019 & & 0.265 & & 0.661 \\
\hline Negative & 78.3 & & 34.9 & & 51.8 & \\
\hline Positive & 42.4 & & 40.0 & & 40.4 & \\
\hline pT stage & & 0.078 & & 0.041 & & 0.210 \\
\hline pT1 & 80.1 & & 41.9 & & 56.4 & \\
\hline pT2-4 & 51.2 & & 31.3 & & 42.0 & \\
\hline pN stage & & 0.260 & & 0.623 & & 0.221 \\
\hline pNo-1 & 75.0 & & 38.6 & & 52.7 & \\
\hline $\mathrm{pN} 2-3$ & 53.9 & & 33.3 & & 43.2 & \\
\hline MFI (mo) & & 0.795 & & 0.756 & & 0.611 \\
\hline$\leq 35$ & 58.5 & & 44.7 & & 52.6 & \\
\hline$>35$ & 70.4 & & 31.2 & & 41.9 & \\
\hline Solitary BM & & 0.852 & & 0.007 & & 0.011 \\
\hline Yes & 66.2 & & 47.8 & & 61.6 & \\
\hline No & 69.6 & & 18.1 & & 26.0 & \\
\hline EBM & & 0.562 & & 0.114 & & 0.235 \\
\hline Yes & 53.3 & & 39.9 & & 22.2 & \\
\hline No & 67.5 & & 16.7 & & 52.4 & \\
\hline RT dose $\left(\mathrm{Gy}_{10}\right)$ & & 0.036 & & 0.002 & & 0.079 \\
\hline$<50$ & 51.1 & & 20.2 & & 41.7 & \\
\hline$\geq 50$ & 86.7 & & 69.0 & & 61.9 & \\
\hline WLRT & & 0.895 & & 0.007 & & 0.005 \\
\hline Yes & 67.7 & & 50.0 & & 61.3 & \\
\hline No & 63.5 & & 20.0 & & 32.1 & \\
\hline MRTI (mo) & & 0.780 & & 0.078 & & 0.984 \\
\hline$\leq 1$ & 71.7 & & 42.2 & & 47.7 & \\
\hline$>1$ & 59.1 & & 32.0 & & 50.2 & \\
\hline Palliative chemotherapy & & 0.763 & & 0.551 & & 0.945 \\
\hline Yes & 70.1 & & 41.7 & & 45.8 & \\
\hline No & 65.6 & & 34.8 & & 50.0 & \\
\hline Palliative endocrinologic therapy & & 0.994 & & 0.689 & & 0.271 \\
\hline Yes & 64.4 & & 35.7 & & 52.8 & \\
\hline No & 71.3 & & 34.9 & & 43.7 & \\
\hline
\end{tabular}

LC, local control; DPFS, distant progression-free survival; OS, overall survival; HER2, human epidermal growth factor receptor 2; MFI, metastasis-free interval; BM, bone metastasis; EBM, extrabony metastasis; RT, radiation therapy; Gy ${ }_{10}$ biological equivalent dose in Gy with $\alpha / \beta=10$; WLRT, whole-lesion RT; MRTI, metastasis-to-RT interval.

favorable prognostic factor with marginal significance $(p=$ 0.055) for the LC of lesions where RT was applied (Table 3). Distant progression occurred in 34 patients. The median DPFS after the initial metastasis was 23 months (95\% confidence interval, 10.04 to 35.96 months). The 3-year and 5-year DPFS rates were $36.8 \%$ and $24.5 \%$, respectively. Pathologic T stage of the primary tumor $(p=0.041)$, solitary BM $(p=0.007)$, higher RT dose $(p=0.002)$, and WLRT ( $p=0.007)$ were determined to be significant factors for longer DPFS after the initial metastasis by univariate analysis. A shorter MRTI ( $\leq 1$ month) tended to be associated with improved DPFS; however, this trend was not significant ( $p=0.078$ ) (Table 2 ). On multivariate 
Table 3. Multivariate analysis

\begin{tabular}{|c|c|c|c|c|c|c|}
\hline \multirow{2}{*}{ Variable } & \multicolumn{2}{|c|}{$\mathrm{LC}$} & \multicolumn{2}{|c|}{ DPFS } & \multicolumn{2}{|c|}{ OS } \\
\hline & $\mathrm{HR}$ & $\mathrm{p}$-value & $\mathrm{HR}$ & p-value & $\mathrm{HR}$ & $p$-value \\
\hline Age ( $<40$ vs. $\geq 40 \mathrm{yr}$ ) & - & - & 0.747 & 0.554 & - & - \\
\hline HER2 (negative vs. positive) & 0.200 & 0.072 & 1.830 & 0.281 & - & - \\
\hline Hormone receptor (negative vs. positive) & - & - & - & - & 7.558 & 0.001 \\
\hline pT stage (pT1 vs. pT2-4) & 0.394 & 0.161 & 0.770 & 0.573 & 0.780 & 0.528 \\
\hline pN stage (pNo-1 vs. pN2-3) & 0.785 & 0.785 & 0.663 & 0.390 & 0.322 & 0.019 \\
\hline $\mathrm{MFI}(\leq 35 \mathrm{vs} .>35 \mathrm{mo})$ & 1.054 & 0.937 & 0.656 & 0.335 & - & - \\
\hline Solitary BM (yes vs. no) & 1.677 & 0.612 & 0.637 & 0.408 & 0.256 & 0.015 \\
\hline EBM (yes vs. no) & 3.602 & 0.297 & 1.834 & 0.34 & 1.548 & 0.504 \\
\hline RT dose (<50 vs. $\geq 50 \mathrm{~Gy}_{10}$ ) & 5.874 & 0.055 & 2.547 & 0.098 & 1.528 & 0.427 \\
\hline WLRT (yes vs. no) & 0.754 & 0.698 & 1.129 & 0.806 & 0.411 & 0.052 \\
\hline MRTI (mo) $(\leq 1$ vs. $>1)$ & 0.926 & 0.920 & 0.458 & 0.122 & - & - \\
\hline Palliative chemotherapy (yes vs. no) & 0.848 & 0.874 & 0.614 & 0.390 & 0.497 & 0.134 \\
\hline Palliative endocrinologic therapy (yes vs. no) & 1.135 & 0.922 & 0.601 & 0.346 & 0.834 & 0.731 \\
\hline
\end{tabular}

LC, local control; DPFS, distant progression-free survival; OS, overall survival; HR, hazard ratio; HER2, human epidermal growth factor

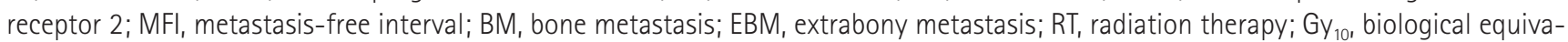
lent dose in Gy with $\alpha / \beta=10$; WLRT, whole-lesion RT; MRTI, metastasis-to-RT interval.

analysis, however, none of above was identified as independent favorable prognostic factors for DPFS (Table 3).

\section{Survival analysis}

The 2-year and 5-year OS rates were $85.2 \%$ and $49 \%$, respectively. Univariate analysis revealed that patients had significantly better survival when the metastasis was solitary BM $(p=0.011)$, and when they had WLRT $(p=0.005)$ (Table 2). A higher RT dose tended to be associated with improved OS, however, not significantly $(p=0.079)$. Positive HR status, negative HER2 status, lower pathologic $T$ and $N$ stages of primary disease, and the lack of extrabony metastasis were factors associated with better survival, but not significantly so as well. Neither palliative chemotherapy nor palliative endocrinologic therapy affected survival significantly in the present study. On multivariate analysis (Table 3), positive HR status ( $p=0.001)$, and solitary BM ( $p=0.015)$ were identified as independent favorable prognostic factors for the survival of patients with EOMBC. WLRT was found to be a favorable prognostic factor for survival with marginal significance ( $p$ $=0.055$ ). A low pathologic nodal stage was an independent favorable prognostic factor according to multivariate analysis $(p=0.011)$, although this result was not obtained by univariate analysis.

\section{Subgroup analysis}

We next analyzed the outcomes in the group of patients with solitary BM $(n=31)$. The 3-year and 5 -year tumor $L C$ rates of this subgroup were $70.6 \%$ and $66.2 \%$, respectively. HER2negative status and high RT dose were significantly associated with improved LC in univariate analysis (Table 4). The 2-year and 5-year DPFS rates of the subgroup were 58.8\% and 35.9\%, respectively. Univariate analysis revealed high RT dose, WLRT, and shorter MRTI ( $\leq 1$ month) as significant factors associated with improved DPFS rates (Table 4). The 2-year and 5-year OS rates were $96.7 \%$ and $61.6 \%$, respectively. WLRT was the only significant factor for improved survival in univariate analysis ( $p$ $=0.031$ ) (Table 4).

\section{Discussion and Conclusion}

Systemic treatment is a mainstay for managing MBC. However, since the concept of $\mathrm{OM}$ was proposed, various studies over the last decade have reported the benefits of local modalities for various metastatic cancers, including $O M B C$, in $L C$ of metastatic lesion, progression-free survival (PFS) and even OS. Milano et al. [10] reported 2-year and 4-year LC rates of $67 \%$ and $60 \%$, respectively for patients who underwent SABR for OMBC metastatic lesions. Rusthoven et al. $[17,18]$ and Lee et al. [19] also reported prospective data of SABR for patients with metastatic cancer, including patients with breast cancer. They reported LC rates at 1 and 2 years of 100\% and $90 \%$, respectively, for lung metastases with RT of 48 to 60 Gy. They also reported an LC rate at 1 year of $71 \%$ for hepatic metastasis. In the present study, the 3-year and 5-year tumor LC rates were $69.6 \%$ and $66.1 \%$, respectively. Our outcome is 
Table 4. Univariate analysis of solitary bone metastasis subgroup

\begin{tabular}{|c|c|c|c|c|c|c|}
\hline \multirow{2}{*}{ Variable } & \multicolumn{2}{|c|}{$5-y r$ LC } & \multicolumn{2}{|c|}{$3-y r$ DPFS } & \multicolumn{2}{|c|}{$5-y r$ OS } \\
\hline & $\%$ & $p$-value & $\%$ & $p$-value & $\%$ & $p$-value \\
\hline HER2 & & 0.005 & & 0.851 & & 0.326 \\
\hline Negative & 77.4 & & 37.1 & & 69.8 & \\
\hline Positive & 31.3 & & 25.0 & & 37.5 & \\
\hline RT dose $\left(\mathrm{Gy}_{10}\right)$ & & 0.016 & & 0.018 & & 0.127 \\
\hline$<50$ & 45.1 & & 29.4 & & 52.9 & \\
\hline$\geq 50$ & 91.7 & & 75.0 & & 74.6 & \\
\hline WLRT & & 0.119 & & 0.035 & & 0.031 \\
\hline Yes & 74.4 & & 57.0 & & 66.2 & \\
\hline No & 43.8 & & 25.0 & & 50.0 & \\
\hline MRTI (mo) & & 0.160 & & 0.023 & & 0.466 \\
\hline$\leq 1$ & 80.8 & & 58.9 & & 65.7 & \\
\hline$>1$ & 47.0 & & 35.7 & & 57.1 & \\
\hline Palliative chemotherapy & & 0.691 & & 0.519 & & 0.535 \\
\hline Yes & 60.0 & & 49.5 & & 60.0 & \\
\hline No & 68.5 & & 40.0 & & 61.9 & \\
\hline Palliative endocrinologic therapy & & 0.439 & & 0.185 & & 0.658 \\
\hline Yes & 59.6 & & 44.0 & & 62.0 & \\
\hline No & 78.8 & & 55.6 & & 60.0 & \\
\hline
\end{tabular}

LC, local control; DPFS, distant progression-free survival; OS, overall survival; HER2, human epidermal growth factor receptor 2; RT, radiation therapy; Gy ${ }_{10}$, biological equivalent dose in Gy with $\alpha / \beta=10$; WLRT, whole-lesion RT; MRTI, metastasis-to-RT interval.

comparable or superior to those of other studies. This result is especially, striking because local modalities were applied with curative intent with ablative RT doses in the previous studies, whereas in our study, local treatments were performed in the palliative setting with non-ablative RT dose. However, a higher RT dose $\left(\geq 50 \mathrm{~Gy}_{10}\right)$ was, still, a favorable factor for $\mathrm{LC}$ in our study. This finding implies that dose escalation for local RT of OMBC might result in better $L C$ of metastatic lesions.

Median DPFS duration of the current study was 23 months, and the 3-year and 5-year DPFS rates were $36.8 \%$ and $24.5 \%$, respectively. Previous studies reported that $30 \%$ to $40 \%$ of OMBC patients who were treated with local modality first and achieved no evidence of disease status showed distant progression within 3 months after the local therapy, and eventually $50 \%$ to $80 \%$ of the patients demonstrated the progression to MBC within 2 years [20]. Our result is comparable with those of other studies. These results imply that the subgroup of patients diagnosed as OMBC could have other lesions which were undetectable clinically, and systemic therapies such as chemotherapy or endocrinologic therapy could be beneficial for treatment of clinically diagnosed EOMBC. However, systemic therapies for EOMBC were not associated with improved DPFS significantly in our study. Multivariate analysis revealed that no significant factor was associated with improved DPFS, although univariate analysis showed that factors related to local RT such as higher RT dose, WLRT and early RT (MRTI $\leq 1$ month) were associated to improved DPFS. In the contrary to MBC, previous reports for outcomes of systemic therapies for OMBC were limited, because randomized controlled trials on efficacy of those for patients with OMBC are only small-scale studies $[20,21]$. Our result, however, could be explained by confounding effects of systemic therapies to DPFS. Further large-scale randomized studies are required to investigate the efficacy of systemic therapies for patients with EOMBC. Nonetheless, the correlation between those factors related to local RT and a longer DPFS still supports Hellman's proposal. This finding implies that early local intervention for EOMBC, i.e., before the metastatic tumors undergo significant genetic changes that may enable additional distant metastases, could prevent further distant progression. Various studies have examined the series of genetic changes required for tumor cells to acquire metastatic facilities. Chiang and Massague [22] proposed a model in which the initiation of metastasis involves processes such as tumor invasion and angiogenesis, whereas the progression of metastasis involves vascular remodeling and immune evasion, in addition to other processes. Throughout this process, genes such as RHOC, VEGF, MET, FGFR, and IL11 play important roles in each step of metastasis. Virulence of tumor cells emerges from the activation of these relevant 
genes that lead to organ-specific metastasis $[23,24]$. Therefore, we hypothesize that early and high-dose local RT eradicates nonvirulent tumor cells, before the significant genetic activation and acquisition of virulence in metastatic tumor cells, thereby leads to longer DPFS.

Various reports show benefits in OS as well as LC and PFS of local modalities for patients with OMBC. Milano et al. [10] reported 2-year and 4-year OS rates of 74\% and $59 \%$, respectively, for patients who underwent SABR for OMBC metastatic lesions. Other studies have reported that surgical resections for metastatic lesions in the lung or liver are associated with improved OS [25-28]. In these studies, the 5-year OS rates of patients with MBC who underwent resection of metastatic lesions in the lung and liver were reported to be $38.5 \%-53 \%$ and $34 \%-61 \%$, respectively. Although these studies exhibited a certain degree of selection bias with respect to the surgical candidates and they were retrospective studies, local modalities for MBC appear to be associated with improved survival for patients with MBC. In the current study, the 2-year and 5-year OS rates were 85.2\% and $49 \%$, respectively. This result is comparable to those of other studies, even though our patients received palliative RT with an insufficient dose for a curative aim. This might be explained by the fact that the majority of patients in the current study had bone-only metastasis which is known to be a factor associated with improved survival compared with visceral metastasis [29]. In our study, both univariate and multivariate analyses revealed that solitary BM was a significant prognostic factor for longer survival, and that is consistent with other studies [30-32]. WLRT was a significant and marginally significant prognostic factor for longer OS in univariate, and multivariate analysis, respectively. Murabito et al. [33] reported that the median OS of patients with MBC who received complete resection of pulmonary metastasis was higher than that of patients who received incomplete resection. Although the clinical outcomes of WLRT versus nonWLRT have not yet been compared, the results of Murabito et al. [33] and our results imply that local treatments that cover the entire extent of disease may result in better OS outcomes. This conclusion cannot be drawn unequivocally from our data because patients with solitary BM predominated in the WLRT group; therefore, some selection bias was present in this group. Nonetheless, additional analysis of the solitary BM subgroup still identified WLRT as a significant factor for improved OS as well as DFPS. Moreover, all of the patients who received non-WLRT in the solitary BM group $(n=8)$ received RT only after the disease had progressed for some time. Therefore, the extents of these progressed diseases were presumably too broad to be covered by the entire RT field. Furthermore, a short MRTI ( $\leq 1$ month) was correlated with improved survival in the solitary BM subgroup. Therefore, although solitary BM itself is significantly associated with longer survival, even better survival outcomes may be expected in this subgroup by the application of high-dose RT earlier. Ideally, the RT should be applied as soon as possible, while the lesion is still small enough for WLRT to be a viable option. For early intervention of OM lesions, early detection is essential. If diagnosis could be achieved earlier for patients with OM status, longer survival might be achieved and more active local RT could be applied. Therefore, optimal surveillance plans and adequate diagnostic modalities are required.

Despite a number of studies reporting the role of systemic therapies for $\mathrm{MBC}$, there was no significant association between systemic therapies and $O S$ in the current study. Role of systemic therapies for prolonged OS of patients with EOMBC is uncertain unlikely to patients with MBC, as it is informed above. $[20,21]$. However, our study demonstrated confounding effect of systemic therapies to OS, on multivariate analysis in which statistical significance of the association between RTrelated variable, WLRT, and OS disappeared. Therefore, further studies investigating the role of systemic therapies for EOMBC are necessary. MFI was not significantly associated with improved survival in our study, either, although a long MFI has been found to be a favorable factor for improved survival in various studies $[8,28,29]$. This finding might be due to its small sample size and potential selection bias, since the study included only patients with $\mathrm{EOMBC}$ who received RT at a single institute.

This study did have several limitations. First, it is a retrospective study from a single institute. Second, the sample size was not sufficient to guarantee the results. Finally, some heterogeneity existed in the systemic therapies. Specifically, various chemotherapeutic and endocrinologic regimens were used; the parameters of these regimens may have affected the OS and PFS. Therefore, it is insufficient to tell that there is no association between the systemic therapies and prognosis of patients with EOMBC. During the period in which EOMBC was diagnosed in the sample group, trastuzumab was not covered by the national health insurance program and thus no patients received target therapy. Trastuzumab, however, is now currently used to treat patients with HER2-positive breast cancer. Therefore, further studies are needed to investigate the outcomes of those patients who have been treated with trastuzumab. 
In conclusion, high-dose RT that is performed early, i.e., while WLRT is still an option, has the potential to improve PFS and $\mathrm{OS}$ of patients with EOMBC.

\section{Conflict of Interest}

No potential conflict of interest relevant to this article was reported.

\section{References}

1. Chia SK, Speers $C H$, D'yachkova $Y$, et al. The impact of new chemotherapeutic and hormone agents on survival in a population-based cohort of women with metastatic breast cancer. Cancer 2007;110:973-9.

2. O'Shaughnessy J. Extending survival with chemotherapy in metastatic breast cancer. Oncologist 2005;10 Suppl 3:20-9.

3. Sakamoto Y, Yamamoto J, Yoshimoto M, et al. Hepatic resection for metastatic breast cancer: prognostic analysis of 34 patients. World J Surg 2005;29:524-7.

4. Pagani O, Senkus E, Wood W, et al. International guidelines for management of metastatic breast cancer: can metastatic breast cancer be cured? J Natl Cancer Inst 2010;102:456-63.

5. Hellman S, Weichselbaum RR. Oligometastases. J Clin Oncol 1995;13:8-10.

6. Resection of the liver for colorectal carcinoma metastases: a multi-institutional study of indications for resection. Registry of hepatic metastases. Surgery 1988;103:278-88.

7. Gadd MA, Casper ES, Woodruff JM, McCormack PM, Brennan MF. Development and treatment of pulmonary metastases in adult patients with extremity soft tissue sarcoma. Ann Surg 1993;218:705-12.

8. Corbin KS, Hellman S, Weichselbaum RR. Extracranial oligometastases: a subset of metastases curable with stereotactic radiotherapy. J Clin Oncol 2013;31:1384-90.

9. Friedel G, Pastorino U, Ginsberg RJ, et al. Results of lung metastasectomy from breast cancer: prognostic criteria on the basis of 467 cases of the International Registry of Lung Metastases. Eur J Cardiothorac Surg 2002;22:335-44.

10. Milano MT, Zhang $H$, Metcalfe SK, Muhs AG, Okunieff P. Oligometastatic breast cancer treated with curative-intent stereotactic body radiation therapy. Breast Cancer Res Treat 2009;115:601-8.

11. Dellas K. Does radiotherapy have curative potential in metastatic patients? The concept of local therapy in oligometastatic breast cancer. Breast Care (Basel) 2011;6:363-8.

12. Patchell RA, Tibbs PA, Walsh JW, et al. A randomized trial of surgery in the treatment of single metastases to the brain. $\mathrm{N}$ Engl J Med 1990;322:494-500.
13. Andrews DW, Scott CB, Sperduto PW, et al. Whole brain radiation therapy with or without stereotactic radiosurgery boost for patients with one to three brain metastases: phase III results of the RTOG 9508 randomised trial. Lancet 2004; 363:1665-72.

14. Sperduto PW, Kased N, Roberge D, et al. Summary report on the graded prognostic assessment: an accurate and facile diagnosis-specific tool to estimate survival for patients with brain metastases. J Clin Oncol 2012;30:419-25.

15. Lee SS, Ahn JH, Kim MK, et al. Brain metastases in breast cancer: prognostic factors and management. Breast Cancer Res Treat 2008;111:523-30.

16. Eisenhauer EA, Therasse P, Bogaerts J, et al. New response evaluation criteria in solid tumours: revised RECIST guideline (version 1.1). Eur J Cancer 2009;45:228-47.

17. Rusthoven KE, Kavanagh BD, Cardenes $H$, et al. Multiinstitutional phase I/II trial of stereotactic body radiation therapy for liver metastases. J Clin Oncol 2009;27:1572-8.

18. Rusthoven KE, Kavanagh BD, Burri SH, et al. Multi-institutional phase I/II trial of stereotactic body radiation therapy for lung metastases. J Clin Oncol 2009;27:1579-84.

19. Lee MT, Kim JJ, Dinniwell $R$, et al. Phase I study of individualized stereotactic body radiotherapy of liver metastases. J Clin Oncol 2009;27:1585-91.

20. Kobayashi T, Ichiba T, Sakuyama T, et al. Possible clinical cure of metastatic breast cancer: lessons from our 30-year experience with oligometastatic breast cancer patients and literature review. Breast Cancer 2012;19:218-37.

21. Rauschecker $H$, Clarke M, Gatzemeier W, Recht A. Systemic therapy for treating locoregional recurrence in women with breast cancer. Cochrane Database Syst Rev 2001;(4):CD002195.

22. Chiang AC, Massague J. Molecular basis of metastasis. N Engl J Med 2008;359:2814-23.

23. Kang $Y$, Siegel PM, Shu W, et al. A multigenic program mediating breast cancer metastasis to bone. Cancer Cell 2003;3:537-49.

24. Minn AJ, Gupta GP, Siegel PM, et al. Genes that mediate breast cancer metastasis to lung. Nature 2005;436:518-24.

25. Tanaka $F, L i M$, Hanaoka $N$, et al. Surgery for pulmonary nodules in breast cancer patients. Ann Thorac Surg 2005;79:1711-4.

26. Pocard M, Pouillart $P$, Asselain $B$, Salmon R. Hepatic resection in metastatic breast cancer: results and prognostic factors. Eur J Surg Oncol 2000;26:155-9.

27. Rena 0 , Papalia $E$, Ruffini $E$, et al. The role of surgery in the management of solitary pulmonary nodule in breast cancer patients. Eur J Surg Oncol 2007;33:546-50.

28. Vlastos G, Smith DL, Singletary SE, et al. Long-term survival after an aggressive surgical approach in patients with breast cancer hepatic metastases. Ann Surg Oncol 2004;11:869-74.

29. Largillier R, Ferrero JM, Doyen J, et al. Prognostic factors in 1,038 women with metastatic breast cancer. Ann Oncol 2008; 
19:2012-9.

30. Ahn SG, Lee HM, Cho SH, et al. Prognostic factors for patients with bone-only metastasis in breast cancer. Yonsei Med J 2013;54:1168-77.

31. Domchek SM, Younger J, Finkelstein DM, Seiden MV. Predictors of skeletal complications in patients with metastatic breast carcinoma. Cancer 2000;89:363-8.
32. Koizumi M, Yoshimoto M, Kasumi F, Ogata E. Comparison between solitary and multiple skeletal metastatic lesions of breast cancer patients. Ann Oncol 2003;14:1234-40.

33. Murabito $M$, Salat A, Mueller MR. Complete resection of isolated lung metastasis from breast carcinoma results in a strong increase in survival. Minerva Chir 2000;55:121-7. 